\title{
Bevacizumab May Overcome Clinical Tumor Drug Resistance to Irinote- can Based Chemotherapy in Patients with Metastatic Colorectal Cancer: Case Report
}

\author{
J.M. Zekri*,1 and M.Z. Abubacker ${ }^{2}$ \\ ${ }^{I}$ Department of Oncology, King Faisal Specialist Hospital and Research Centre, P.O. BOX 40047, Jeddah 21499, \\ MBC\#: J-64, Kingdom of Saudi Arabia \\ ${ }^{2}$ Department of Radiology, King Faisal Specialist Hospital and Research Centre, P.O. BOX 40047, Jeddah 21499, \\ MBC\#: J-64, Kingdom of Saudi Arabia
}

\begin{abstract}
Introduction: In the management of patients with metastatic colorectal cancer (mCRC) failing anti-cancer agents/regimens are substituted by others. There is no data in the literature showing that combining 2 failing agents/regimens can induce tumor response.

Case: We report a case with mCRC who failed first line XELOX/bevacicumab and second line FOLFIRI. However, the tumour responded to third line FOLFIRI/bevacizumab.

Conclusion: Retreatment with bevacizumab in combination with a failing chemotherapy regimen is a possible treatment option after exhaustion of all standard treatments. This approach seems to be effective in overcoming clinical tumor drug resistance to irinotecan based chemotherapy
\end{abstract}

Keywords: Metastatic, colorectal cancer, chemotherapy, angiogenesis \& bevacizumab.

\section{INTRODUCTION}

In the last decade, development of novel therapies that target biological pathways improved outcome of patients with metastatic colorectal cancer $(\mathrm{mCRC})$. The current accepted standard practice in management of $\mathrm{mCRC}$ is to substitute ineffective agents with others that have not proven to be ineffective sequentially in the same patient. The exception to this standard is in patients with established irinotecan refractory disease where cetuximab is introduced and irinotecan is restarted. This is based on the clinical observation that cetuximab may overcome resistance to irinotecan [1].

Bevacizumab, a humanized monoclonal antibody binds to and neutralizes vascular endothelial growth factor. Bevacizumab improved overall survival and progression free survival in first and second line settings when added to chemotherapy [2-4]. Here: we report a case refractory to first line oxaliplatin based chemotherapy combined with bevacizumab and second line irinotecan based chemotherapy. However, the tumour responded to combination of the 2 failing regimens, namely irinotecan based chemotherapy and bevacizumab.

\section{CASE}

57 years old man diagnosed in December 2008 with sigmoid adenocarcinoma. He underwent rectosigmoid resection. Pathological examination showed poorly differentiated

*Address correspondence to this author at the Department of Oncology, King Faisal Specialist Hospital and Research Centre, P.O. BOX 40047, Jeddah 21499, MBC\#: J-64, Kingdom of Saudi Arabia; Tel: 009662667 7777, Ext 64348, Secretary 64010; Fax: 0096626677777 (Ext 64030); Mobile: 00966 540659819; E-mail: jmzekri@ hotmail.com adenocarcinoma with focal signet ring pattern and mucin secretion. Two out of 7 lymph nodes (LNs) were positive for metastatic carcinoma. K-ras mutation was present at codon 12.

Postoperative CT scan showed multiple non resectable liver metastases, largest in segment 7 measuring $3.3 \times 2.7 \mathrm{~cm}$ (Fig. 1).

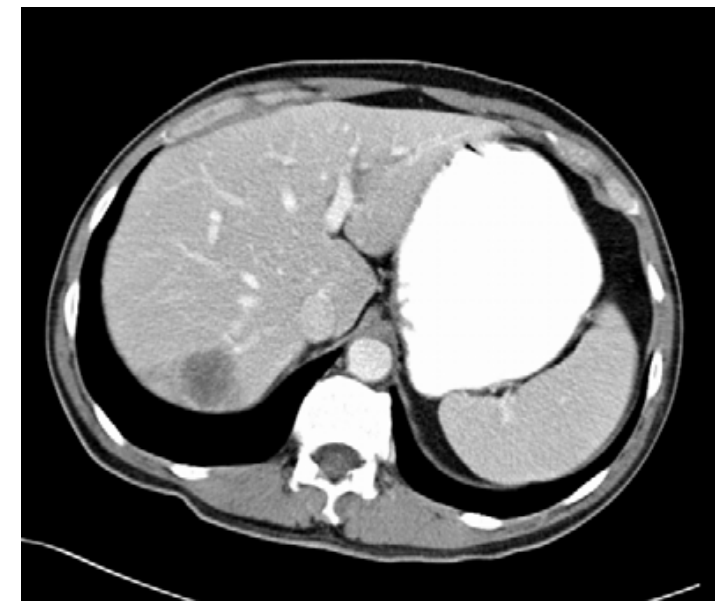

Fig. (1). Initial baseline CT demonstrated multiple lesions with a target lesion (arrow) in segment VII measuring 33mm longest diameter.

On 5/1/2009 he started palliative XELOX/bevacizumab (XELOX-B). He received 3 cycles of XELOX-B. CEA (carcinoembryonic antigen) continued to rise (Graph 1) and a CT on $3 / 3 / 2009$ confirmed increase in size of liver metastases. 


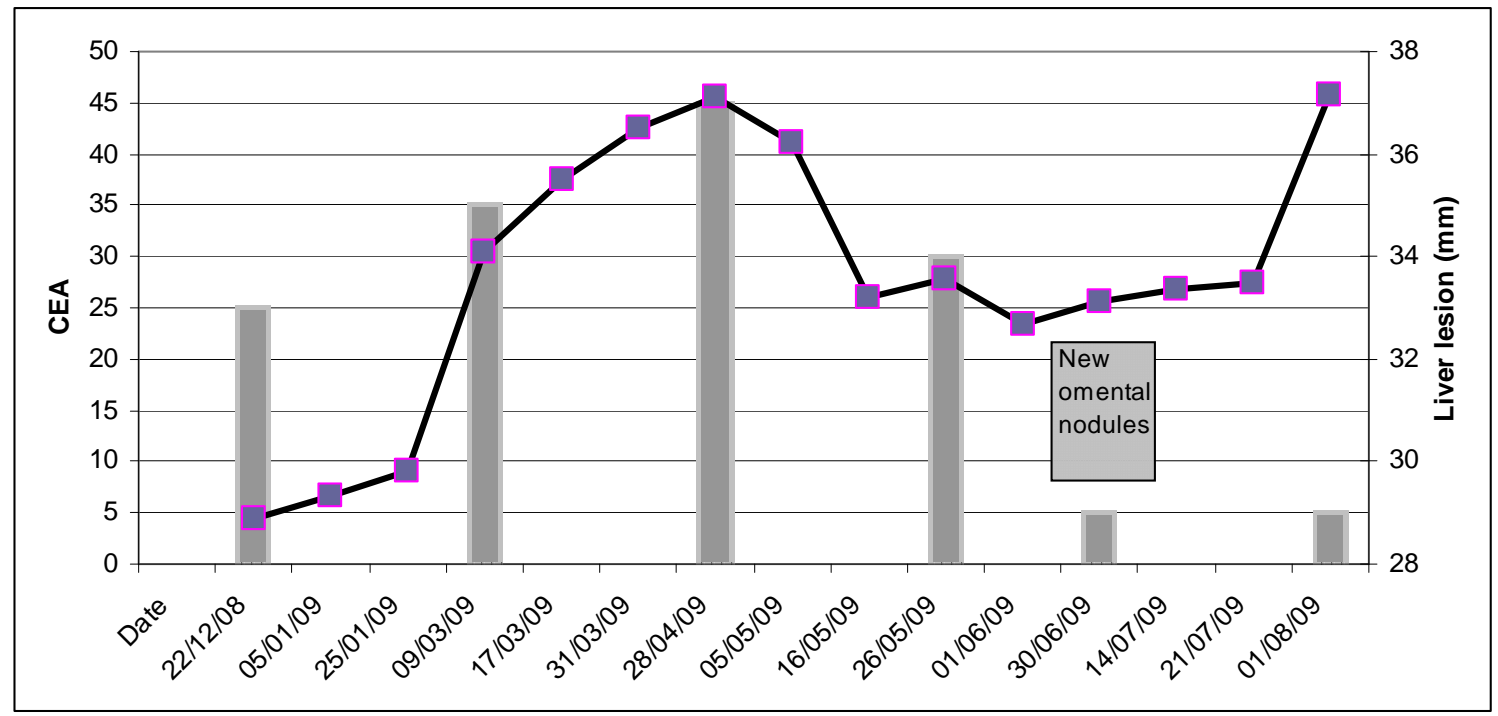

Graph 1. Plot of CEA and longest diameter of target liver lesion over time. (Line represents CEA and columns represent size of liver lesion).

On 10/3/2009 he started FOLFIRI. He received 4 cycles of FOLFIRI. CEA continued to rise (Graph 1) and a CT on 22/4/2009 demonstrated subtle increase in size of liver metastases (Fig. 2).

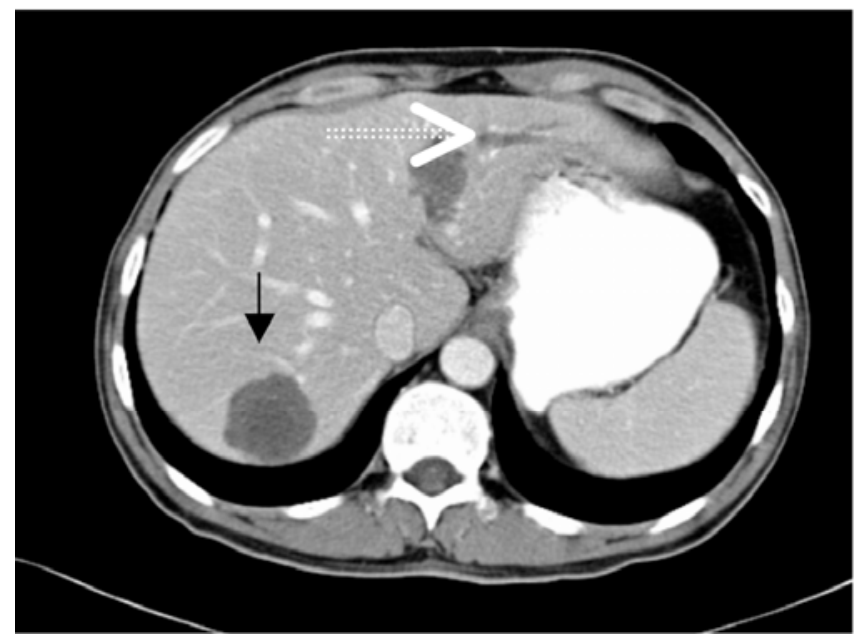

Fig. (2). Follow up CT reveals further increase in size of the liver lesions with the target lesion now measuring $37 \mathrm{~mm}$ (black arrow), in longest diameter and new intra-hepatic duct dilatation (white dotted arrow).

On 29/4/2009 bevacizumab was added to FOLFIRI (FOLFIRI-B). CEA showed decline (Graph 1). CT on 27/5/2009 showed reduction in size of liver metastases (Fig. 3). FOLFIRI-B was continued. On 1/7/2009 he received the fifth cycle of FOLFIRI-B. On 4/7/2009 he was admitted with increasing abdominal pain. CT on 5/7/2009 showed further reduction in size of liver metastases (Fig. 4a) and the presence of omental disease in the form of fat stranding and nodularity with in the omental fat under the anterior abdominal wall (Fig. 4b). These radiological findings correspond to the site of abdominal pain and palpable lower abdominal thickening, induration, nodularity and tenderness on palpation.

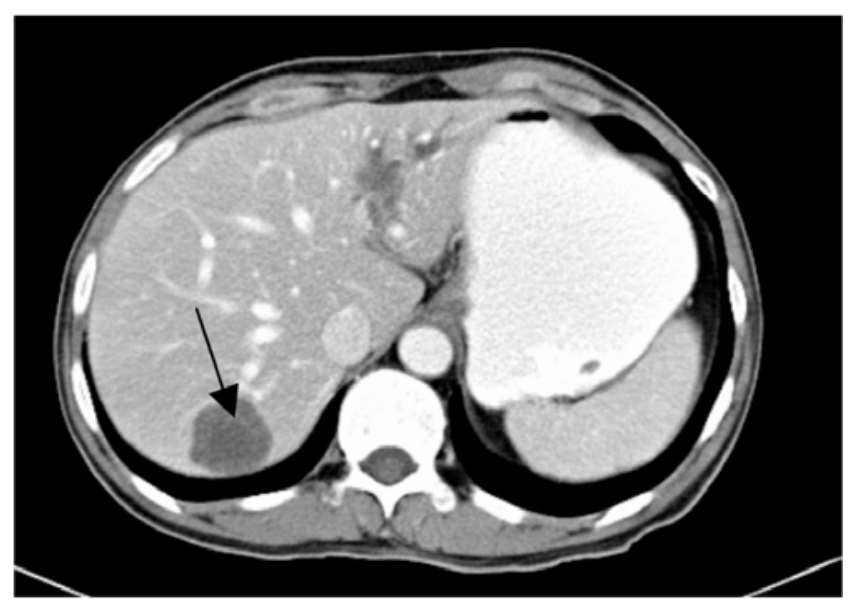

Fig. (3). Liver lesions have now decreased minimally in size with the target lesion now measuring $34 \mathrm{~mm}$ (arrow) in the longest diameter.

FOLFIRI-B was discontinued. Clinical, CEA and radiological parameters continued to confirm progressive disease (Graph 1). The patient died on 7/9/2009.

\section{DISCUSSION}

Our case presents an observation of response to combination 2 anti-cancer agents/regimens after each one of them have failed when used alone or in combination with another regimen. Bevacizumab failed to induce response when combined with XELOX in the first line setting. FOLFIRI also failed in the second line setting. However, tumour response was observed when 2 failing components were combined together namely Bevacizumab and FOLFIRI.

The approach of combining failing agents is unusual in the management of mCRC and hence our observation is also unusual. This observation may lead to the speculation that bevacizumab can overcome resistance to irinotecan. This concept have been investigated and proven to be effective with cetuximab [1]. However, this concept was not clinically tested with bevacizumab. In our case, it is possible that 

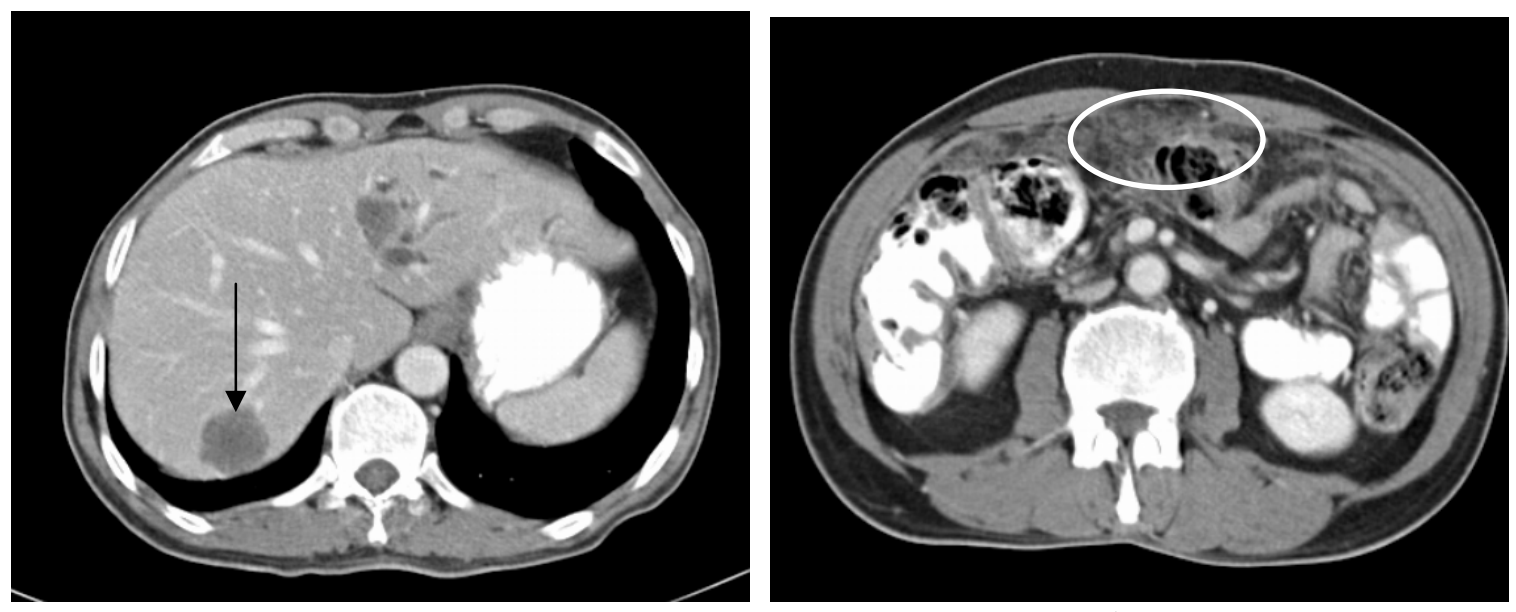

एயm

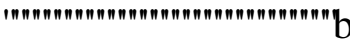

Fig. (4). a) Further reduction in the size of the longest diameter of the target liver lesion (arrow) to 29 mm and b) New omental nodularity noted (circled).

Bevacizumab modified dysfunctional tumor vasculature thereby improving tumor perfusion and consequently delivery of systemic chemotherapy to the tumor. This phenomenon has been documented in tumour xenografts. Neuroblastoma xenografts treated with bevacizumab showed progressive decrease in their microvessel density. Assessment of the tumor microenvironment revealed a rapid sustained decrease in both tumor vessel permeability and tumor interstitial fluid pressure, whereas intratumoral perfusion was improved. Penetration of chemotherapy was improved by as much as $81 \%$ when given 1 to 3 days after bevacizumab [5].

In the literature, there are 2 clinical observations that are only partly similar to our observation and may support the concept of ability of bevacizumab overcome resistance to chemotherapy. First; the observation that third line or later bevacizumab containing chemotherapy regimens induced responses after progression on oxaliplatin and irinotecan based regimens [6-8]. Unlike our case, patients in these reports were bevacizumab naïve. Second; the observation of improved survival after changing the chemotherapy and continuing the use of bevacizumab beyond first tumor progression compared to changing the chemotherapy and stopping bevacizumab in a large observational study [9]. Unlike our case, patients in this report received second chemotherapy regimen different from the first line.

Halama et al. treated 5 five patients who were heavily pretreated and already had received cetuximab (and in two cases also bevacizumab). These patients received a chemotherapeutic regimen consisting of irinotecan, cetuximab and bevacizumab. The combination of these two antibodies with irinotecan induced marked tumor response in 4 out of 5 patients [10]. More recently a large randomized phase III trial confirmed that adding cetuximab to XELOX-B in the first line setting resulted in significantly shorter progressionfree survival and inferior quality of life compared to XELOX-B alone [11]. Thus the approach of combining both antibodies is not recommended.

Our patient progressed on all appropriate standard treatment options for his disease. After discussion with the patient he agreed to undergo treatment with combination of 2 failing regimens/agents on the ground that he has not been exposed to both of them simultaneously. We observed radiological response and progression free survival (PFS) lasting for 9 weeks. The duration of PFS is acceptable in third line setting and after failure of all standard options.

In our case and upon progression on first line therapy the target liver lesion showed central density changes (Fig. 2). This radiological change is well recognized and often termed radiologically as central necrosis [12] In view of increasing lesion size and rising CEA this change does not represent response to treatment.

Evaluation of tumor response to targeted therapies remains controversial. Most physicians and investigators agree that traditional radiologic size changes are insufficient and probably inappropriate in this setting. Most investigated is the role of ${ }^{18} \mathrm{~F}$-FDG PET in assessing response of gastrointestinal stromal tumors (GIST) to imatinib. ${ }^{18} \mathrm{~F}$-FDG PET is superior to $\mathrm{CT}$ in predicting early response to therapy in recurrent or metastatic GIST [13]. There is some evidence that ${ }^{18}$ F-FDG PET may predict the pathologic response to Bevacizumab based therapy. Investigators correlated pathological complete response (pCR) to CT and ${ }^{18} \mathrm{~F}$-FDG PET response after treatment of liver metastases from colorectal cancer with bevacizumab and irinotecan. ${ }^{18}$ F-FDG PET correctly predicted pCR in $70 \%$ vs. $35 \%$ by CT [14]. Our patient did not undergo ${ }^{18}$ F-FDG PET studies due to (a) unavailability of this technique locally and (b) CT tumor size changes were parallel to CEA changes at all times.

In summary, this case demonstrates that continuing or retreatment with bevacizumab in combination with a failing chemotherapy regimen is a possible treatment option after exhaustion of all standard treatments. Clinically, this approach seems to be effective in overcoming tumor drug resistance to irinotecan based chemotherapy. Investigating this approach in phase II clinical trials is recommended.

\section{ABBREVIATIONS AND ACRONYMS}

B $=$ Bevacizumab.

CEA $=$ Carcinoembryonic antigen. 


$$
\begin{aligned}
& \text { FOLFIRI }=\text { Folinic acid, fluorouracil and irinotecan } \\
& \text { mCRC }=\text { Metastatic colorectal cancer } \\
& \text { XELOX }=\text { Capecitabine and oxaliplatin }
\end{aligned}
$$

\section{REFERENCES}

[1] Cunningham D, Humblet Y, Siena S, et al. Cetuximab monotherapy and cetuximab plus irinotecan in irinotecan-refractory metastatic colorectal cancer. N Engl J Med 2004; 351(4): 337-45.

[2] Hurwitz H, Fehrenbacher L, Novotny W, et al. Bevacizumab plus irinotecan, fluorouracil, and leucovorin for metastatic colorectal cancer. N Engl J Med 2004; 350(23): 2335-42.

[3] Kabbinavar FF, Hambleton J, Mass RD, Hurwitz HI, Bergsland E, Sarkar S. Combined analysis of efficacy: the addition of bevacizumab to fluorouracil/leucovorin improves survival for patients with metastatic colorectal cancer. J Clin Oncol 2005; 23(16): 3706.

[4] Giantonio BJ, Catalano PJ, Meropol NJ, et al. Bevacizumab in combination with oxaliplatin, fluorouracil, and leucovorin (FOLFOX4) for previously treated metastatic colorectal cancer: results from the Eastern Cooperative Oncology Group Study E3200.; Eastern Cooperative Oncology Group Study E3200. J Clin Oncol 2007; 25(12): 1539-44.

[5] Dickson PV, Hamner JB, Sims TL, et al. Bevacizumab-induced transient remodeling of the vasculature in neuroblastoma xenografts results in improved delivery and efficacy of systemically administered chemotherapy. Clin Cancer Res 2007; 13(13): 3942-50.

[6] Shitara K, Munakata M, Muto O, Sakata Y. Metastatic rectal cancer responding to third-line therapy employing bevacizumab after failure of oxaliplatin and irinotecan: case report. Jpn J Clin Oncol 2008; 38(7): 493-6.

[7] Kang BW, Kim TW, Lee JL, et al. Bevacizumab plus FOLFIRI or FOLFOX as third-line or later treatment in patients with metastatic colorectal cancer after failure of 5-fluorouracil, irinotecan, and oxaliplatin: a retrospective analysis. Med Oncol 2009; 26(1): 32-7.

[8] Chen HX, Mooney M, Boron M, et al. Phase II multicenter trial of bevacizumab plus fluorouracil and leucovorin in patients with advanced refractory colorectal cancer: an NCI Treatment Referral Center Trial TRC-0301. J Clin Oncol 2006; 24(21): 3354-60.

[9] Grothey A, Sugrue MM, Purdie DM, et al. Bevacizumab beyond first progression is associated with prolonged overall survival in metastatic colorectal cancer: results from a large observational cohort study (BRiTE). J Clin Oncol 2008; 26(33): 5326-34.

[10] Halama N, Herrmann C, Jaeger D, Herrmann T. Treatment with cetuximab, bevacizumab and irinotecan in heavily pretreated patients with metastasized colorectal cancer. Anticancer Res 2008; 28 (6B): 4111-5.

[11] Tol J, Koopman M, Cats A, et al. Chemotherapy, bevacizumab, and cetuximab in metastatic colorectal cancer. N Engl J Med 2009; 360(6): 563-72.

[12] Sica GT Ji H, Ros PR. CT and MR imaging of hepatic metastases. AJR Am J Roentgenol 2000; 174: 691-698.

[13] Gayed I, Vu T, Iyer R, et al. The Role of ${ }^{18}$ F-FDG PET in Staging and Early Prediction of Response to Therapy of Recurrent Gastrointestinal Stromal Tumors. J Nucl Med 2004; 45(1): 17-21.

[14] Goshen E, Davidson T, Zwas ST, Aderka D. PET/CT in the evaluation of response to treatment of liver metastases from colorectal cancer with bevacizumab and irinotecan. Technol Cancer Res Treat 2006; 5(1): 37-43. 